\title{
Primer caso de EXIT (tratamiento exútero intraparto) en el Hospital Universitario San Ignacio: presentación de caso
}

First EXIT (exuterus intapartum treatment) in San Ignacio University Hospital: Case Report

Carolina Mateus

Médica otorrinolaringóloga, Pontificia Universidad Javeriana, Bogotá, Colombia. Unidad de Otorrinolaringología, Departamento de Cirugía,

Hospital Universitario San Ignacio, Colombia ORCID: https://orcid.org/0000-0001-7946-7671

Ana María Acosta ${ }^{a}$

Médica otorrinolaringóloga. Profesora asistente de Otorrinolaringología de la Facultad de

Medicina, Universidad Javeriana, Unidad de Otorrinolaringología, Departamento de Cirugía,

Hospital Universitario San Ignacio, Colombia

ORCID: https://orcid.org/0000-0002-3959-6601

María M. Baquero

Médica otorrinolaringóloga, Pontificia Universidad

Javeriana, Bogotá, Colombia. Unidad de

Otorrinolaringología, Departamento de Cirugía,

Hospital Universitario San Ignacio, Colombia

ORCID: https://orcid.org/0000-0003-4732-6529

PaOla Gómez

Médica anestesióloga, Pontificia Universidad Javeriana,

Bogotá, Colombia. Departamento de Anestesia,

Hospital Universitario San Ignacio, Colombia ORCID: https://orcid.org/0000-0001-6000-3922

JUAN D. LALINDE

Médico ginecobstetra, Pontificia Universidad Javeriana,

Bogotá, Colombia. Departamento de Ginecología y

Obstetricia, Hospital Universitario San Ignacio,

Colombia

ORCID: https://orcid.org/0000-0001-8704-3684

Javier E. Cubillos

Staff Anesthesiologist, London Health Sciences Center. Assistant professor of the Department of Anesthesia and Perioperative Medicine, Western University, London, Ontario, Canadá. ORCID: https://orcid.org/0000-0002-1948-3175
Rodolfo A. Martínez

Médico ginecobstetra. Especialista en Medicina Materno Fetal. Ginecobstetra de Alto Riesgo. Profesor asistente de la Pontificia Universidad Javeriana,

Bogotá, Colombia. Departamento de Ginecología y Obstetricia, Hospital Universitario San Ignacio ORCID: https://orcid.org/0000-0002-2498-1478

Angélica P. Fajardo

Médica anestesióloga pediatra, Departamento de Anestesia, Hospital Universitario San Ignacio, Bogotá,

Colombia.

ORCID: https://orcid.org/0000-0003-2166-9048

Juan Camilo Ospina Médico otorrinolaringólogo pediatra, Universidad de

British Columbia, Vancouver, Canadá. Profesor asistente de Otorrinolaringología de la Facultad de Medicina, Pontificia Universidad Javeriana, Unidad de Otorrinolaringología, Departamento de Cirugía, Hospital Universitario San Ignacio. ORCID: https://orcid.org/0000-0001-7806-5355

\footnotetext{
a Autora de correspondencia: anamaria.acostar@gmail.com
}

Cómo citar: Mateus C, Acosta AM, Baquero MM, Gómez P, Lalinde JD, Cubillos JE, Martínez RA, Fajardo AP, Ospina JC. Primer caso de EXIT (tratamiento exutero intraparto) en el Hospital San Ignacio: reporte de caso. Univ. Med. 2020;61(3). https://doi.org/10.11144/Javeriana.umed61-3.exit 


\section{RESUMEN}

El artículo es la presentación del primer procedimiento exútero intraparto (EXIT), realizado en el Hospital Universitario San Ignacio y realizado por un grupo interdisciplinario (anestesiología, otorrinolaringología pediátrica, neonatología y ginecología). La ejecución de la estrategia EXIT es poco frecuente. Su objetivo es asegurar la vía aérea del neonato durante el parto cuando existe riesgo de obstrucción, dada por compresión secundaria a masas cervicales o mediastinales, malformaciones congénitas craneofaciales o de la vía aérea. Esta estrategia busca disminuir el riesgo de mortalidad del neonato. El caso corresponde al de una mujer primigestante de 33 años, con edad gestacional de 38 semanas y diagnóstico a la semana 25 de lesión quística cervical en el feto. Durante la atención del parto se aseguró intraparto la vía aérea del neonato con intubación orotraqueal y, a su vez, se disminuyeron los riesgos de morbimortalidad de la madre. En este caso, se trataba de una malformación vascular linfática macroquística. El objetivo del artículo es destacar que, con una adecuada planeación y diagnóstico prenatal de las malformaciones cervicales, mejora significativamente el pronóstico del paciente, así como sus resultados a corto y largo plazo.

\section{Palabras clave}

EXIT (procedimiento exútero intraparto); malformación cervical congénita; vía aérea.

\footnotetext{
ABSTRACT

The present article is the report of the first Ex Utero Intrapartum (EXIT) procedure, carried out at the San Ignacio University Hospital, by an interdisciplinary group (anesthesiologist, pediatric otolaryngologist, neonatologist and obstetrician). The need for an EXIT strategy is rare. Its objective is to secure the newborn's airway during ¿hildbirth when there is a risk of airway obstruction tha may be caused by secondary compression from cervical or mediastinal masses, congenital craniofacial or airway malformations. The aim of the surgery is to reduce the risk of mortality of the newborn. We present a 33 years old patient with a gestational age of 38 weeks and a diagnosis of cervical cystic lesion in the fetus by gestational week 25. The delivery was performed by securing the neonate's airway with orotracheal intubation. In this case, a vascular macrocystic lymphatic malformation was confirmed. The objective of the article is to emphasize that proper planning and prenatal diagnosis of cervical malformations significantly improves the patient's prognosis, both in the short and the long term results.
}

Keywords

EXIT (ex-uterus intrapartum treatment); congenital cervical malformation, airway.

\section{Introducción}

El procedimiento exútero intraparto (EXIT) está diseñado para asegurar durante el parto la vía aérea del recién nacido, cuando está afectada por masas cervicales (teratomas o malformaciones linfáticas o vasculares), hernia diafragmática congénita con compresión traqueal o síndrome de obstrucción de vía aérea congénita (CHAOS). También está indicado en casos de atresia laríngea, micrognatia severa, enfermedades cardiacas congénitas que requieran adicionalmente oxigenación extracorpórea o teratomas mediastinales que necesiten resección inmediata (1).

Es indispensable el diagnóstico prenatal de estas alteraciones para disminuir el riesgo de mortalidad del recién nacido, por lo que una vez detectada una malformación que pueda afectar la vía aérea del neonato, debe remitirse a un hospital de tercer o cuarto nivel, que cuente con la experticia, junto con estudios imagenológicos para la planeación del procedimiento EXIT. Se requiere un equipo multidisciplinario que incluye ginecobstetra de alto riesgo, anestesiólogo obstetra y pediátrico, otorrinolaringólogo pediatra o cirujano pediatra y neonatólogo. Intraoperatoriamente, debe haber dos equipos quirúrgicos completos: uno para la madre y otro para el recién nacido.

Este procedimiento implica una modificación de la cirugía de cesárea y los objetivos anestésicos difieren de aquellos para esta última; incluso son opuestos. Dentro de sus objetivos está la relajación uterina profunda, la preservación de 
la circulación uteroplacentaria y la inmovilidad y anestesia fetal sin depresión cardiaca $(2,3)$. La hipotonía uterina es esencial para facilitar la extracción del feto y prevenir el desprendimiento de la placenta y, de esta manera, garantizar el flujo uteroplacentario durante la intervención $(4,5)$.

La principal técnica usada es la anestesia general balanceada; sin embargo, hay casos que han descrito anestesia neuroaxial, sumada a uso endovenoso de medicamentos tocolíticos $(4,6)$.

Durante la anestesia balanceada, estos objetivos anestésicos se logran a través de dosis altas de anestésico halogenado, usualmente de 2 a 3 MAC en el momento de la histerotomía, ya que a estas dosis los anestésicos inhalados inhiben la contracción uterina. En caso de ser insuficiente, se complementa con la administración de medicamentos tocolíticos por vía endovenosa, como nitroglicerina, sulfato de magnesio o terbutalina $(2,5)$. La consecuencia hemodinámica de esta estrategia es la vasodilatación sistémica y la disminución de la resistencia vascular periférica con hipotensión secundaria, por lo cual se requiere una adecuada reanimación hídrica que puede incluir el uso de cristaloides y coloides, al igual que de vasopresores, como la fenilefrina (2). Así, se logra mantener la presión de la perfusión uterina y preservar la circulación uteroplacentaria. De igual forma, a través de esta circulación, se administra la anestesia fetal, que en el caso de ser ineficaz se suma a la administración directa de opioides y relajantes neuromusculares al feto, ya sea por vía intravenosa o por vía intramuscular, logrando la inmovilidad y la anestesia necesaria para llevar a cabo los procedimientos requeridos en el feto antes del pinzamiento del cordón umbilical (4).

El principal riesgo durante el procedimiento es el sangrado mayor como consecuencia de la relajación uterina profunda; por ello, una vez pinzado el cordón umbilical, se debe lograr rápidamente un aumento adecuado del tono uterino. Para esto se debe administrar tempranamente oxitocina y disminuir a menos de uno la concentración del MAC del anestésico inhalado. Dentro de las opciones está descrito el uso de desflurano, por ser un anestésico inhalado de rápida titulación, y el paso de una técnica anestésica balanceada a una intravenosa total, al igual que el uso de otros uterotónicos, como misoprostol o metilergonovina, en caso de persistencia de atonía uterina $(4,7)$.

Consideraciones adicionales incluyen la inducción de secuencia rápida y el uso preventivo de un procinético y regulador del $\mathrm{pH}$ gástrico, por tratarse de una población en riesgo de broncoaspiración. Se debe monitorear con línea arterial ante la posibilidad de compromiso hemodinámico y requerimiento de medicamentos vasoactivos, y la administración de una infusión de cristaloides tibios en la cavidad uterina para preservar el volumen uterino y prevenir el enfriamiento fetal $(2,4,7)$.

En cuanto a la técnica para la cesárea, la incisión de elección es, por lo general, transversal baja, a menos que la posición anterior de la placenta requiera una histerectomía posterior, cuando se realizaría una incisión mediana. Justo antes del comienzo de la cesárea, es necesario ubicar con precisión la implantación de la placenta con un ultrasonido y así evitar su sección al momento de la histerotomía (8). Asimismo, mediante la ecografía, podemos conocer la posición de la cabeza del feto y el cuello para evitar la manipulación excesiva del feto después de la histerotomía.

Además, para manipular adecuadamente el feto, a veces es necesario descomprimir las masas quísticas, lo cual se puede antes de la cesárea. Después de la histerotomía, el mantenimiento del volumen uterino es uno de los pasos más importantes en un EXIT. Esto se hace para disminuir la probabilidad de la contracción uterina y desprendimiento de la placenta y mantener continua la transferencia de oxígeno materno-fetal. La infusión de una solución de lactato de Ringer tibia después de la histerotomía permite mantener el volumen uterino y evita la compresión del cordón umbilical. Asimismo, la exposición limitada del feto durante el EXIT ayuda a mantener el volumen uterino y la temperatura fetal. Solo la cabeza, el cuello y los hombros y una extremidad superior están expuestos mientras se mantiene el resto del feto dentro de la cavidad uterina. 
Es limitada la evaluación de la vía aérea y los tejidos circundantes del cuello solo mediante ultrasonido, por lo que el uso de imágenes por resonancia magnética de alta resolución (3 Tesla) antes del nacimiento pueden brindar una mejor visualización de la relación de la masa con la vía aérea y ayudar a predecir qué pacientes están en mayor riesgo de padecer obstrucción de su vía área y, por lo tanto, necesiten un procedimiento EXIT. No se han demostrado efectos nocivos en los recién nacidos sometidos a resonancia magnética en el periodo prenatal y neonatal (9).

El otorrinolaringólogo pediatra y el anestesiólogo pediatra deben estar preparados para asegurar la vía aérea con intubación endotraqueal, laringoscopia, broncoscopia rígida, traqueostomía u oxigenación extracorpórea. En algunos casos será necesaria la resección de masas pulmonares o mediastinales en ese mismo momento. Es indispensable el acompañamiento de cirugía pediátrica.

Al elegir el procedimiento EXIT, es importante brindar una explicación amplia y clara a la madre, y cuando sea pertinente, a los familiares, acerca de los riesgos, las complicaciones y los posibles desenlaces fatales.

Se ha reportado una mortalidad del 8-10\% en neonatos llevados a EXIT por masas cervicales gigantes, comparado con un $57 \%$ en los que no fueron manejados bajo este procedimiento (7). Por lo que es fuertemente recomendado, y cada vez con mayores indicaciones, en los hospitales de alto nivel de complejidad.

El objetivo de presentar este caso clínico es documentar el primer procedimiento EXIT realizado en el Hospital Universitario San Ignacio.

\section{Caso clínico}

El caso corresponde a una mujer de 33 años de edad, remitida de Bucaramanga (Colombia), con embarazo de 38 1/7 semanas por ecografía de primer trimestre, en seguimiento por medicina de alto riesgo con diagnóstico de quiste cervical fetal en estudio, diagnosticado en la semana 25. Una resonancia magnética nuclear encontró en el feto una lesión quística de contornos irregulares y bien definidos con algunos tabiques hipointensos que afectaban los tejidos blandos superficiales y los profundos de la región mandibular izquierda, con placenta con implantación alta corporal anterior, sin pérdida de la interfase de la unión entre el miometrio y la placenta (figura 1).

\section{Figura 1.}

Resonancia magnética nuclear materna. Se observa un feto con malformación quística en región mandibular izquierda

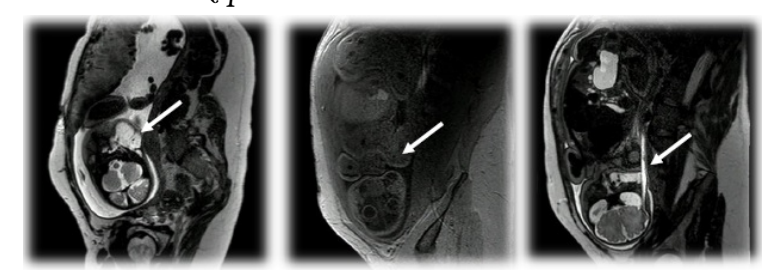

Se revisó el caso de la paciente en una junta médica interdisciplinaria, en la cual se decidió que la mujer se beneficiaría del procedimiento EXIT para asegurar la vía aérea del feto en el nacimiento.

Todo el equipo quirúrgico (obstetra de alto riesgo, anestesiólogo materno y pediátrico, otorrinolaringólogo pediatra y neonatólogo) se encontraba presente y preparado para el ingreso de la gestante a la sala de cirugía (figura 2).

\section{Figura 2.}

Disposición de la sala de cirugía durante procedimiento EXIT

\section{SALA DE CIRUGÍA}

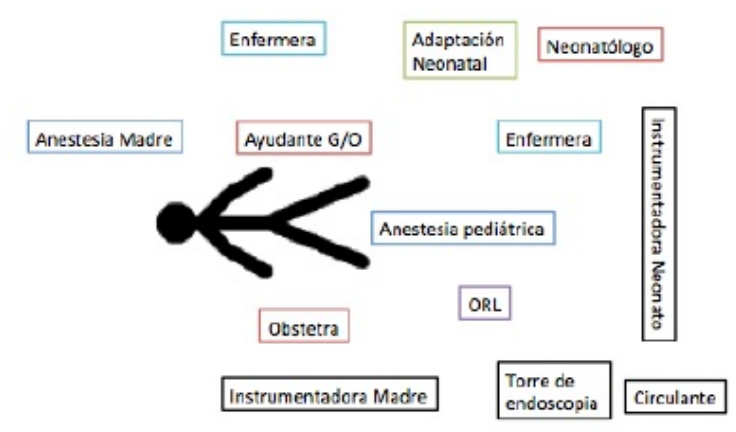

Se prepararon todos los insumos y equipos que potencialmente serían utilizados durante la cirugía, como laringoscopios, broncoscopios, cánulas de succión, cánulas de traqueostomía, 
equipo de anestesia materna y neonatal y equipo de adaptación neonatal (figura 3).

Figura 3.

Instrumental para la vía aérea del recién nacido

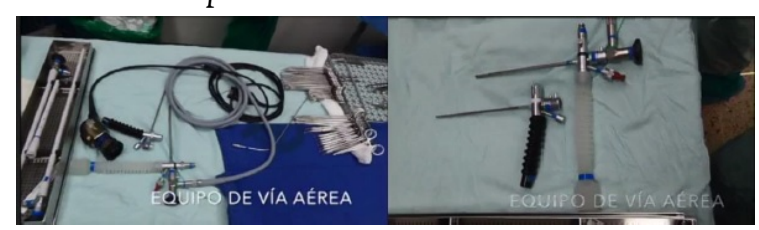

La paciente había sido premedicada con metoclopramida y ranitidina 30 minutos antes del procedimiento. Se le colocó monitoría básica, preoxigenación con máscara facial y oxígeno al 100 \%. Se realizó inducción de secuencia rápida e intubación orotraqueal. Se canalizaron dos venas periféricas con Jelco $14 \mathrm{G}$ y una línea arterial radial derecha. El mantenimiento anestésico se hizo con una técnica balanceada con sevoflurano entre 2 y 4,8 vol \% y remifentanilo en infusión a $0,1-0,2 \mu \mathrm{g} / \mathrm{kg} / \mathrm{min}$.

El equipo de obstetricia de alto riesgo inició la cesárea, y para el momento de la histerotomía se optimizó la relajación uterina con bolos de 50 a $100 \mu \mathrm{g}$ de nitroglicerina intravenosa y se inició una infusión de cristaloides tibios en la cavidad uterina. Una vez se ejecutó la histerotomía arciforme de concavidad superior, se extrajo la cabeza del neonato, se rotó y se colocó en posición de decúbito supino con la cabeza y el miembro superior derecho por fuera del útero, colocando en la mano el sensor del oxímetro de pulso. En ese momento, anestesiología pediátrica llevó a cabo una laringoscopia directa con hoja Miller 0, e intubó con tubo orotraqueal 3.0 sin balón, asegurando la vía aérea. Durante la intervención fetal no fue necesario administrar medicamentos adicionales, ya que se logró una adecuada anestesia fetal vía transplacentaria. Se extrajo completamente el recién nacido, se realizó triple pinzamiento del cordón umbilical y se inició la ventilación con bolsa de ambú (figuras 4 y 5$)$.

\section{Figura 4.}

Intubación neonatal intraparto

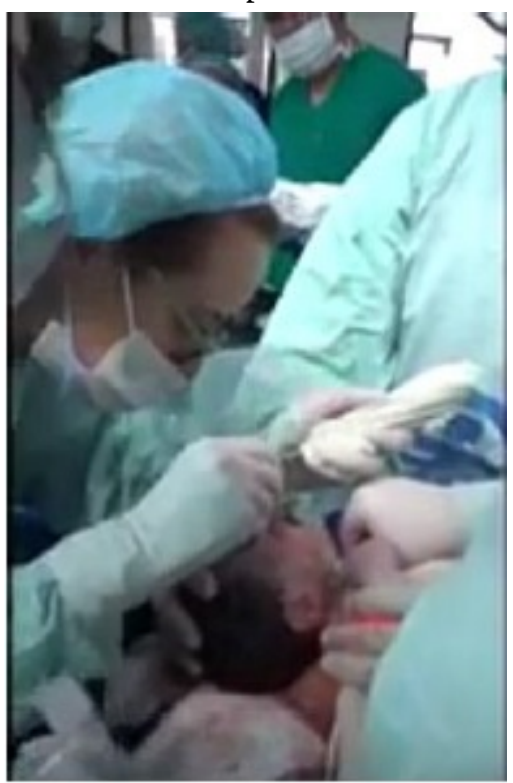

\section{Figura 5.}

Luego de la intubación orotraqueal se realizó el pinzamiento del cordón umbilical y la adaptación neonatal

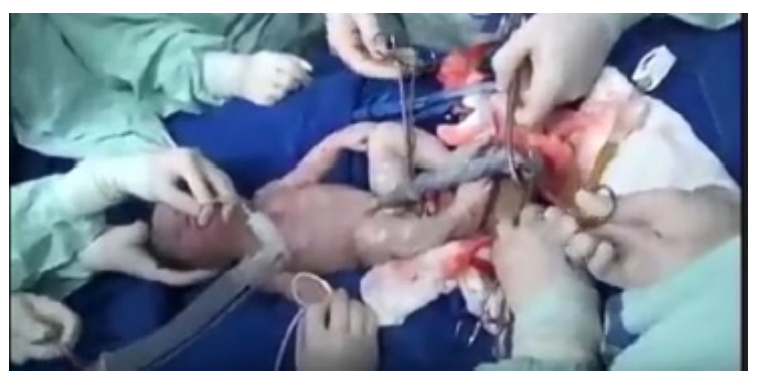

Las cifras tensionales de la paciente se mantuvieron entre $100-120$ y $60-80 \mathrm{~mm} \mathrm{Hg}$ usando infusión de $0,5 \mu \mathrm{g} / \mathrm{kg} / \mathrm{min}$ de fenilefrina y reanimación con cristaloides.

El recién nacido se trasladó a la mesa de neonatología y pediatría, al tiempo que se continuó con el proceso de adaptación neonatal, ventilando con presión positiva. Se verificó tono muscular, frecuencia cardiaca, frecuencia respiratoria, saturación y expansión torácica. En conjunto con anestesiología pediátrica se decidió extubarlo, ya que en el examen físico se observó una masa compresible en el ángulo mandibular izquierdo que se extendía $5 \mathrm{~cm}$ hacia el punto de contacto distal, de apariencia clínica sugestiva 
de malformación linfática sin compresión de la vía aérea (figura 6). El neonato se extubó sin complicaciones y presentó llanto normal, sin presencia de estridor ni dificultad respiratoria.

Figura 6.

Malformación vascular linfática macroquística en región submandibular izquierda

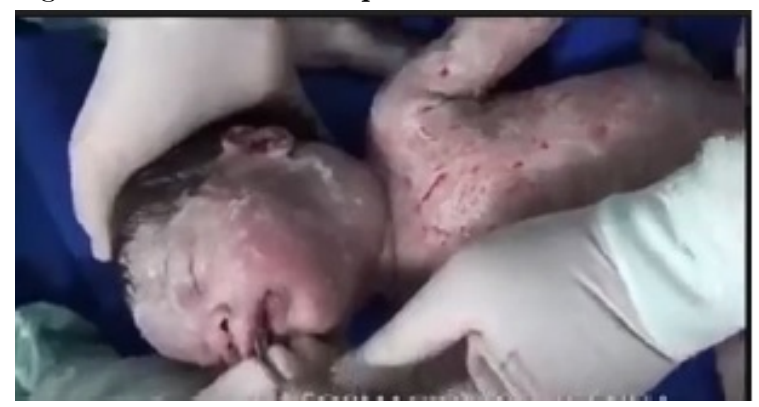

Simultáneamente, tras la extracción completa del feto y la placenta, y una vez pinzado el cordón umbilical, se iniciaron las maniobras de recuperación del tono uterino, se cambió de técnica anestésica a una total intravenosa con propofol y remifentanilo en infusión, se suspendió la infusión de fenilefrina, se aumentó el flujo de gases frescos para favorecer la rápida eliminación del anestésico inhalado y se administró oxitocina y una dosis de metilergonovina vía intramuscular. Posterior a ello se logró un adecuado tono uterino. Adicionalmente, se administró un gramo de ácido tranexámico. La cesárea se terminó de forma rutinaria.

Al finalizar el procedimiento se bloqueó el cuadrado lumbar bilateral, guiado por ecografía con $20 \mathrm{ml}$ de bupivacaína al 0,25\% con epinefrina y $20 \mathrm{ml}$ de lidocaína al $2 \%$ sin epinefrina.

El procedimiento se llevó a cabo sin complicaciones y las metas anestésicas se cumplieron. El sangrado quirúrgico fue aproximadamente de $500 \mathrm{ml}$. En total, se administraron $3000 \mathrm{ml}$ de cristaloides. La paciente se extubó al final del procedimiento y se trasladó a la unidad de cuidados posanestésicos y a piso ese mismo día. Fueron satisfactorios tanto la evolución materna como del recién nacido.

Por parte de neonatología se realizó la adaptación neonatal sin complicaciones y el paciente se trasladó a hospitalización en cuidado intermedio. Posteriormente, fue valorado por radiología intervencionista para manejar la malformación linfática.

El neonato fue llevado a salas de angiografía, donde bajo anestesia general, utilizando una guía ecográfica con transductor lineal, mediante aguja catéter, se realizó un acceso controlado a las formaciones quísticas de la lesión. Posteriormente, se drenó contenido líquido por aspiración, con la consiguiente disminución del volumen de la malformación. Luego, previa quistografía, se administró controladamente, bajo fluoroscopia, el agente esclerosante intralesional (bleomicina), a fin de evitar el paso a circulación sistémica sin complicaciones con resolución de masa cervical (figura 7).

\section{Figura 7.}

A) Quistografía.

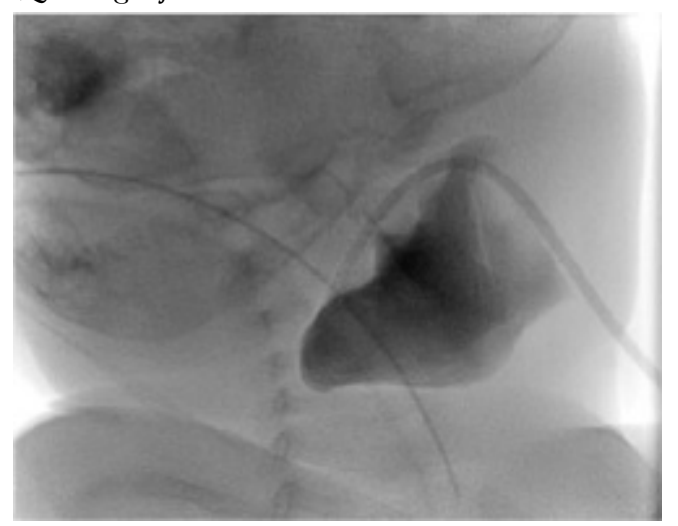

Figura 7.

B) Contenido macroscópico líquido

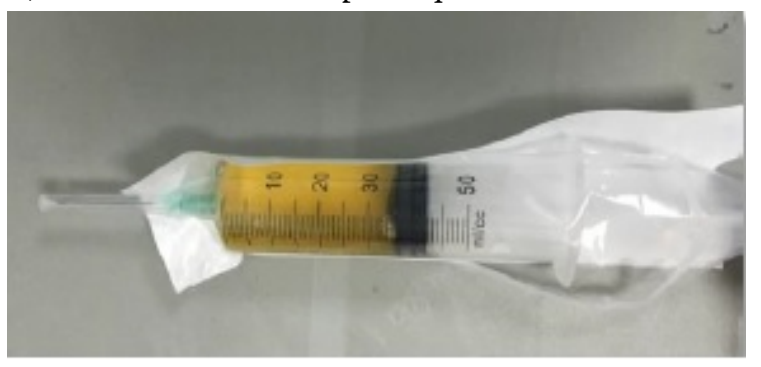

\section{Discusión}

El diagnóstico oportuno de una malformación cervical potencialmente letal en el feto disminuye considerablemente el riesgo de mortalidad perinatal. Por medio de una ecografía prenatal 
es posible detectar diferentes anormalidades. Idealmente, debe complementarse con una resonancia magnética nuclear fetal para establecer la afectación de la vía aérea, con el fin de evitar la irradiación que generan otras técnicas, al tiempo que se obtiene una valoración más precisa de la anatomía.

Estos neonatos siempre se deben manejar en un hospital de tercer o cuarto nivel de complejidad, donde se cuente con un equipo multidisciplinario que permita tratarlo de manera integral y que esté preparado para identificar y resolver posibles complicaciones. Este equipo debe estar conformado por ginecobstetra de alto riesgo, otorrinolaringólogo pediatra, cirujano pediatra, anestesiólogo pediatra y anestesiólogo obstetra, quienes deben estar preparados y coordinados para ofrecer tanto a la madre como al neonato el mejor desenlace posible.

Durante el EXIT tienen igual importancia la disponibilidad del equipo humano descrito como el del instrumental quirúrgico y equipos de apoyo para realizar los procedimientos planeados. Debe contarse con un equipo quirúrgico para el soporte de la madre y otro equipo para el soporte del neonato. Cuando el grupo de vía aérea se enfrente a un primer caso de EXIT, previamente debía de haber preparado con meticulosidad cada fase del proceso con asignación de roles y responsabilidades. Debe de haber un líder que coordine todas las acciones. Para casos posteriores, idealmente es preciso contar con un protocolo de manejo para estandarizar el procedimiento. La revisión de listas de chequeo es imprescindible. El equipo está obligado a prever los diferentes escenarios clínicos y tomar las medidas pertinentes, incluyendo los escenarios adversos como pérdida de vía aérea, necesidad de traqueostomía, hipovolemia materna, muerte, entre otros.

Una vez se establece una vía aérea segura, el neonato debe ser revaluado para confirmar el diagnóstico y planear la estrategia del manejo definitivo médico-quirúrgico de la lesión. En nuestro caso, se trataba de un neonato con una malformación vascular linfática macroquística, que fue manejada satisfactoriamente con bleomicina intralesional, un agente esclerosante.
Las malformaciones linfáticas tienen una incidencia de 1 en 2000-4000 nacidos vivos y el $75 \%$ se presenta en la región cervicofacial, como lo fue en este paciente. Según su tamaño, se clasifican en macroquísticas $(>2 \mathrm{~cm}$, únicas o múltiples), microquísticas $(<2 \mathrm{~cm})$ o mixtas. Se caracterizan por ser lesiones expansivas sin disminuir su tamaño con el tiempo. Sin embargo, tienen una característica especial que permiten comprimirlas, dado su contenido líquido. El 50 $\%$ se presenta durante el nacimiento y el 80-90 $\%$ se manifiesta a los 2 años de edad. Se caracterizan por ser lesiones pequeñas azulosas con nódulos rojizos, por la presencia de sangrado o inflamación. Cursan con crecimiento gradual y la expansión y compresibilidad son típicas (10).

Aunque estas malformaciones pueden ser diagnosticadas por medio del examen físico, las imágenes diagnósticas como ecografía, Doppler y resonancia nuclear magnética permiten, además de confirmar el diagnóstico, identificar la arquitectura de los quistes y determinar la extensión de la enfermedad. Su patrón característico en la resonancia consiste en realce de tabiques y de la periferia en forma de aros o $\operatorname{arcos}(10)$.

En cuanto al tratamiento de estas malformaciones, no existe un manejo ideal o estándar. Existen reportes de involución esporádica; sin embargo, la mayoría de estas lesiones continúan creciendo con la edad.

La escleroterapia está indicada frecuentemente en las malformaciones linfáticas para lesiones profundas o de difícil acceso quirúrgico. Dentro los agentes más utilizados se encuentran el etanol, la bleomicina, el OK-432, la doxiciclina, entre otros $(11,12)$. Por último, la cirugía se reserva para lesiones remanentes al manejo con escleroterapia o láser (10).

\section{Referencias}

1. Dighe MK, Peterson SE, Dubinsky TJ, Perkins J, Cheng E. EXIT procedure: technique and indications with prenatal imaging parameters for assessment of airway patency. 
Radiographics. 2011;31(2):511-26. htt p://doi.org/10.1148/rg.312105108

2. Garcia P, Olutoye OO, Ivey RT, Olutoye OA. Case scenario: anesthesia for maternal-fetal surgery. The ex utero intrapartum therapy (EXIT) procedure. Anesthesiology. 2011;114(6):1446-52.

3. Oliveira E, Pereira P, Retroz C, Mártires E. Anesthesia for EXIT procedure (ex utero intrapartum treatment) in congenital cervical malformation-a challenge to the anesthesiologist. Braz J Anesthesiol. 2015;65(6):529-33.

4. Sviggum HP, Kodali BS. Maternal anesthesia for fetal surgery. Clin Perinatol. 2013;40(3):413-27.

5. Marwan A, Crombleholme TM. The EXIT procedure: principles, pitfalls, and progress. Semin Pediatr Surg. 2006;15(2):107-15.

6. George RB, Melnick AH, Rose EC, Habib AS. Case series: Combined spinal epidural anesthesia for Cesarean delivery and ex utero intrapartum treatment procedure. Can J Anesth. 2007;54(3):218-22.

7. Laje P, Johnson MP, Howell LJ, Bebbington MW, Hedrick HL, Flake AW, et al. Ex utero intrapartum treatment in the management of giant cervical teratomas. J Pediatr Surg. 2012;47(6):1208-16. https://doi.org/10 $.1016 /$ j.jpedsurg.2012.03.027

8. Hedrick HL. Ex utero intrapartum therapy. Semin Pediatr Surg. 2003;10:190-5.

9. Hubbard AM, Crombleholme TM, Adzick NS. Prenatal MRI evaluation of giant neck masses in preparation for the fetal EXIT procedure. Am J Perinatol. 1998;15:253-7.

10. Cox J, Barlett E, Lee EI. Vascular malformations: a review. Semin Plast Surg. 2014;28(2):25-63.
11. Gresham TR, Friedman AB. Hemangiomas and vascular malformations: current theory and management. Int $\mathrm{J}$ Pediatr. 2012:645678. https://doi.org/10.1155/ 2012/645678

12. Ospina J, Wuesthoff C, Eslava S. EXIT: tratamiento exútero intraparto. Reporte de caso y revisión de la literatura. Rev Colomb Obstet Ginecol. 2012;63(2):155-62. 